\title{
Hybrid OFDM RoF-Based WDM-PON/MMW Backhaul Architecture for Heterogeneous Wireless Networks
}

\author{
Trang Thi Thu Ngo, Thu Anh Pham, Nhan Duc Nguyen, Ngoc The Dang \\ Posts and Telecommunications Institute of Technology, Hanoi, Vietnam \\ Correspondence: Trang Thi Thu Ngo, trangntt1@ptit.edu.vn \\ Communication: received 29 November 2016, revised 7 August 2017, accepted 14 August 2017 \\ Online publication: 7 March 2018, Digital Object Identifier: 10.21553/rev-jec.150 \\ The associate editor coordinating the review of this article and recommending it for publication was Dr. Nguyen Tan Hung.
}

\begin{abstract}
In this paper, a hybrid backhaul architecture, which is based on wavelength-division multiplexing passive optical networks (WDM-PON) and millimeter-wave (MMW) communications, is proposed to deliver orthogonal frequencydivision multiplexing (OFDM) signals in heterogeneous wireless networks. MMW radio-over-fiber (RoF) technique, which combines the advantages of the both optical fiber and wireless communications, is used to simplify the base stations and provide flexibility long reach and high capacity connections. The feasibility of the proposed hybrid backhaul architecture is investigated via the bit-error rate (BER) performance of a downlink under the impacts of fiber nonlinear, wireless fading and noise components including clipping noise, amplifier noise, and photodetector noise. The numerical results obtained from this study help to determine the optimum system parameters such as the optical launched power, modulation index, and amplifier gain so as to minimize the link's BER.
\end{abstract}

Keywords- Orthogonal frequency-division multiplexing, radio over fiber, millimeter-wave RoF, cellular backhaul networks.

\section{INTRODUCTION}

The explosive growth of mobile data traffic and a massive increase in the number of wirelessly interconnected devices are exhausting the capabilities of existing wireless networks. One of the strategies to deal with the shortage of global bandwidth in wireless communications is to increase the working frequency to the millimeter-wave band, which also helps to provide higher capacity to the end users. Therefore, the millimeter-wave band has recently been proposed for future broadband cellular communication networks such as the fifth-generation (5G) mobile networks, which require thousand fold increase in the system capacity and tenfold in spectral efficiency and data rate compared to $4 \mathrm{G}$ mobile networks [1, 2]. However, the disadvantages of MMW frequency bands are the requirements of highly directional beam forming antennas in both mobile devices and base stations and the short distance between transmitting and receiving antennas [1]. Hence, a large number of base stations (BSs) need to be deployed. As a result, they should have small coverage so-called small cells while remote cells are expected to be compact, simple, and energy efficient.

Cellular backhaul is a term usually used to describe connectivity between core network and BSs over a variety of transport media. In 5G heterogeneous wireless cellular architecture, there are, however, two types of BSs, macrocell BS (MBS) and small cell BS (SBS). In a macrocell, one MBS is placed in the center and SBSs are distributed in the macrocell. Accordingly, a new

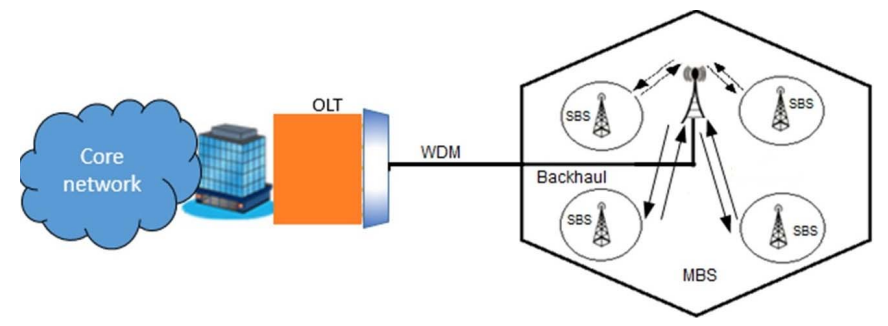

Figure 1. Hybrid RoF based WDM-PON/MMW architecture for heterogeneous wireless networks.

solution of 5G cellular backhaul networks has been proposed in [3]. In this solution, the traffic of small cells is sent from the SBSs to the MBS via MMW backhaul links, and then fiber to the cell (FTTC) links is used to carry the aggregated backhaul traffic from the MBS to the core network.

Practically, to save the infrastructure cost, the optical distributed networks (ODNs) should be shared between cellular backhaul networks and other access networks. The most popular and widely used ODNs are that of passive optical networks (PONs). The use of PONs for cellular backhaul to carry baseband data to/from BSs has been studied and tested according to current standards [4,5]. Although PONs based on time-division multiplexing (TDM-PON) can meet the capacity requirements of cellular backhaul traffic, the scheduler needs to be modified to prioritize backhaul traffic due to asymmetric delay characteristic [5]. An attractive alternative is wavelength-division multiplexing PON (WDM-PON), which can provides 


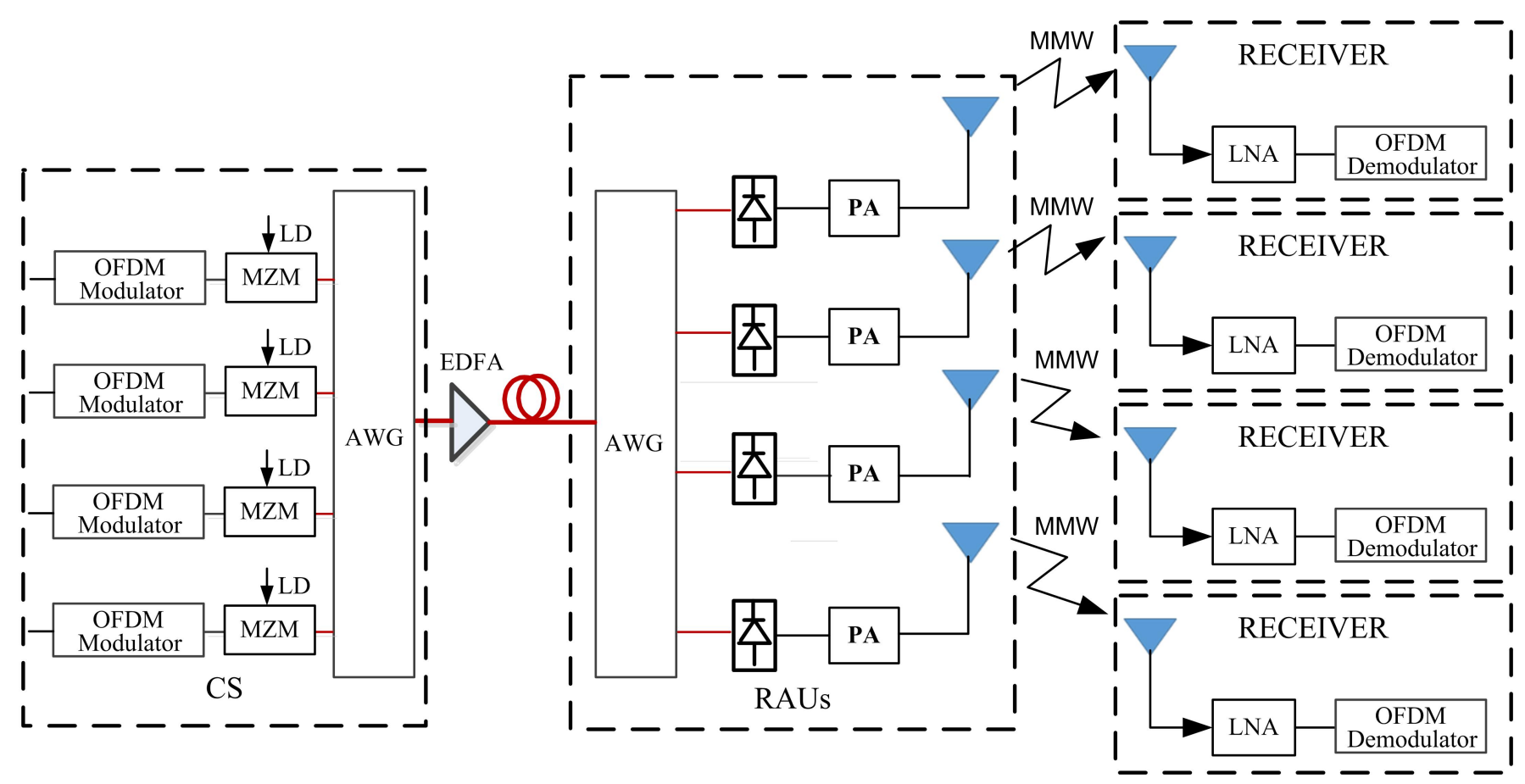

Figure 2. An architecture of hybrid OFDM RoF-based WDM-PON/MMW downlink.

a significant reduction in the number of fibers while supplying a large number of high data-rate channels. On the other hand, radio-over-fiber (RoF) technology, which combines the advantages of the both optical fiber and wireless communications, provides flexibility, long reach, high capacity, low electromagnetic interference, and high immunity to the atmospheric conditions [6, 7]. In RoF-based backhaul networks, complex functions such as modulation/demodulation and up/down-conversion to/from RF frequency are located at the CS while optical fibers in capable of providing high data-rate with low loss are used to distribute the data-modulated RF signals from CS to BSs. RoF is more effective when it is used to deliver orthogonal frequency-division multiplexing (OFDM) signal wireless access networks.

To inherit the advantages from above-mentioned techniques, we therefore propose a hybrid backhaul architecture for heterogeneous wireless networks as shown in Figure 1. In the proposed architecture, the OFDM signal is modulated with MMW carrier at the CS and converted to optical signal before being delivered to MBS via WDM-PON using RoF technique. Next, OFDM RoF signal is sent to SBSs via MMW links. In order to investigate the feasibility of the proposed hybrid backhaul architecture, the performance of an OFDM RoF-based WDM-PON/MMW downlink will be analyzed under the effects of various physical impairments originated from both optical and electrical domain including fiber nonlinear, wireless fading, and other several noise components such as clipping noise, amplifier noise, and photodetector (PD) noise. Bit error rate (BER) of the proposed system will be investigated with different system parameters such as the launched power and modulation index to provide useful information for system design.

The rest of this paper is organized as follows. Section 2 demonstrates the architecture of an OFDM RoFbased WDM-PON/MMW downlink system. Performance analysis will be presented in Section 3. Section 4 demonstrates the numerical results and discussion. Finally, Section 5 concludes the paper.

\section{Hybrid OFDM RoF-BASEd WDM-PON/MMW DOWNLINK}

Figure 2 shows architecture of hybrid OFDM RoF-based WDM-PON/MMW downlink. The downlink consists of four main subsystems, including CS, WDM-PONbased ODN, MBS-located remote antenna unit (RAU), and SBSs-located remote radio heads (RRHs). Considering the requirement of a backhaul connection in terms of capacity, we investigate the WDM-PON with four wavelengths. In addition, this number of wavelengths is enough for us to investigate all the cases of wavelength combinations induced by FWM effect such as degenerate and nondegenerate terms.

At the CS, based on the chosen modulation scheme, e.g., M-QAM, OFDM modulator maps the input data onto complex symbols carried by each subcarrier. As the optical modulator is based on the intensity modulation, the real and positive time domain signal is required. Consequently, Hermitian symmetry and asymmetrically clipped optical (ACO) or DC-biased optical (DCO) solutions are employed. In this study, we choose ACO-OFDM scheme due to its advantage in the reduction of the optical power required for a given data rate. At the OFDM modulator, each OFDM signal is 
modulated on RF MMW subcarrier. Based on subcarrier intensity modulation (SIM) scheme, at Mach-Zehnder modulator (MZM), pre-modulated RF MMW carrier is used to drive the intensity of an optical carrier (at a specific wavelength). In our proposed system, the double sideband (DSB) modulation is used for reducing the cost and simplifying the transmitter. Modulated optical carriers from MZMs are then multiplexed at wavelength multiplexer such as array waveguide grating (AWG) before being transmitted through a fiber. An Erbium-doped fiber amplifiers (EDFA) is used to extend the physical range of PON and reduce the optical power budget. Because EDFA is an active element, therefore it is often placed at the CS to amplify the signal channels to the level required for launching into the fiber link.

In RAU, an AWG is used to de-multiplex the WDM signal into separate optical signals. Each optical signal is directly converted to electrical one thanks to a photodetector (PD). MMW signal from PD is filtered, amplified, and fed to the antenna to transmit to the corresponding RRH via MMW link. At the RRH, the received signal will be amplified by low noise amplifier (LNA). Then, the data is reconstructed by the OFDM demodulator.

\section{Performance Analysis}

In this section, we analyze the impact of physical layer impairments originated from WDM-PON and wireless link including receiver's noise, FWM and wireless fading. Besides FWM, there are actually other nonlinear distortions caused by MZI, photodetectors, power amplifiers, optical fiber (e.g., self-phase mixing and cross-phase mixing). These nonlinear distortions are, however, well investigated in previous studies and they are not the specialized feature of OFDM RoF-based WDM-PON. Therefore, in the scope of this study, we do not take these nonlinear distortions into consideration.

\subsection{Noise Components in OFDM RoF-based WDM-PON}

In this subsection, various noise components of the OFDM RoF-based WDM-PON will be computed, including clipping noise appearing in OFDM modulator $\left(N_{\mathrm{ACO}}\right)$, the amplified spontaneous emission (ASE) noise induced by EDFA ( $\left.N_{\text {EDFA }}\right)$, the noise caused by FWM $\left(N_{\mathrm{FWM}}\right)$, and the noise originated from photodetector $\left(N_{\mathrm{PD}}\right)$. All these noise components are assumed to be independent Gaussian random variables [8-10].

By adding a positive level $\epsilon$ to the ACO-OFDM signals in time domain, we can get the real and positive values for the IM-DD optical channel. Unfortunately, this also causes the clipping noise in the OFDM modulator. The ACO clipping noise power on each wavelength carrier in the $\mathrm{CS}\left(P_{\mathrm{ACO}}\right)$ depends on the optical clipping power ratio (OCPR), i.e., $\eta_{\mathrm{OCPR}}$, as follows $[8,11]$

$$
\eta_{\mathrm{OCPR}}=\frac{\epsilon}{P_{\mathrm{OFDM}}}
$$

$$
\begin{aligned}
P_{\text {ACO }}= & Q^{2}\left(\frac{\eta_{\text {OCPR }}}{\sqrt{2 \pi}}\right)\left(f_{2} \eta_{\text {OCPR }}^{2} \frac{N}{4 \pi(N-1)}\right. \\
& \left.-f_{1} \eta_{\text {OCPR }} \sqrt{\frac{N}{4 \pi(N-1)}}+\frac{f_{0}}{4}-1\right),
\end{aligned}
$$

where $P_{\text {OFDM }}$ is the average optical power of OFDM signal and $f_{i}=\exp \left(p_{i}\right)$ with $p_{i}$ is a polynomial function of $\eta_{\text {OCPR }}[8,11]$. Therefore, the ACO clipping noise in the RAU is written as

$$
N_{\mathrm{ACO}}=\left(R m P_{\mathrm{ACO}} e^{-\alpha L}\right)^{2}
$$

where $R$ is the responsivity of the photodiode, $m$ is the modulation index, $\alpha$ is the attenuation coefficient of the fiber, and $L$ is the fiber length.

In our proposed system, EDFA is used to assure that the power of the signal after the photodiode is high enough for MMW link. EDFA is used to assure that the power of the signal after the photodiode is high enough for MMW link. However, EDFA not only boosts the signal power but also produces ASE noise. The interaction between the existing signals in the fiber creates beat noises remarkably. Among of these, the signal-ASE beat noise and the ASE-ASE beat noise are the dominant elements. In this case, the EDFA has the noise power spectrum density given by [9]

$$
S_{\mathrm{ASE}}=2 \eta_{\mathrm{sp}} \frac{h c}{\lambda}(G-1),
$$

where $G$ is EDFA gain. $\eta_{s p}=N F / 2$ is the spontaneous noise factor, where $N F$ is the noise figure. $h$ and $c$ is Plank constant and the speed of light in vacuum, respectively. $\lambda$ is the wavelength of the optical carrier. We denote $P_{O C}$ as the average power of the optical carrier. The beating noise power in RAU therefore can be obtained as

$$
\begin{aligned}
N_{\mathrm{ASE}}= & 4 R^{2}\left(\left(P_{\mathrm{OFDM}}+P_{\mathrm{OC}}\right) G e^{-\alpha L} S_{\mathrm{ASE}} B_{e} e^{-\alpha L}\right. \\
& \left.+S_{\mathrm{ASE}}^{2}\left(\frac{B_{0}-B_{e}}{2}\right) B_{e} e^{-2 \alpha L}\right)
\end{aligned}
$$

where $B_{e}$ is the electrical bandwidth and $B_{o}$ is the optical bandwidth. In (5), there are two types of beat noise including the signal-ASE beat noise (the 1st term) and the ASE-ASE beat noise (the 2nd term).

FWM is the dominant effect that degrades the performance of the OFDM WDM systems [10, 12]. FWM effect generates a FWM wave, by energy transferring provided by the interaction between three optical waves with equal spacing, called pump waves. For illustration purpose, the optical frequencies of four waves are denoted as $v_{1}, v_{2}, v_{3}$, and $v_{4}$. We also assume that the frequency spacing $\Delta v=v_{i+1}-v_{i}$ is constant. The frequency of the FWM wave, $v_{1}$, is obtained from the frequencies of the three pump waves as $v_{1}=v_{2}+$ $v_{3}-v_{4}$ [13]. Depending on the frequencies of three pump waves, the FWM effect can be categorized into two types: non-degenerated if all pump frequencies are different and degenerated if any two of these frequencies coincide. The contribution of the different pump waves $v_{i}$ on the FWM wave $v_{1}$ depends on the channel spacing between the $i$-th pump wave and the 
FWM wave. It is represented by the walk-off parameter $d_{1, i}=\beta_{1,1}-\beta_{1, i} \approx\left(\frac{D_{c}}{v_{1}}\right) \frac{\left|v_{i}-v_{1}\right|}{v_{1}+\left|v_{i}-v_{1}\right|}$, where $D_{c}$ is the dispersion coefficient of the fiber and $i=2,3,4$. When the optical carrier power is assumed to be much higher than the power of OFDM signal, the FWM waves generated between the three optical carriers or the two optical carriers and one OFDM signal distort the transmitted signal much more seriously than the others. The FWM noise power in the edge channels consists of the power from the degenerate terms $\left(v_{2}=v_{3}\right)$ and the nonsymmetric nondegenerate terms $\left(v_{1} \neq v_{4}\right)$. Due to the presence of the symmetric nondegenerate terms $\left(v_{1}=v_{4}\right)$, the FWM noise power in the central channels is higher. Based on the closed-form expression derived from [10], the FWM noise power for the each channel in RAU can be determined as follows

$$
\begin{aligned}
N_{\mathrm{FWM}}=2 R^{2} e^{-2 \alpha L} L_{\mathrm{eff}} P_{\mathrm{OC}}^{3} P_{\mathrm{OFDM}} G^{4} & \\
& \times \sum_{i=1}^{5} a_{d, i} n_{i}(f)\left(\frac{D \gamma}{3}\right)^{2},
\end{aligned}
$$

where $L_{\text {eff }}=\frac{1-\exp (-\alpha L)}{\alpha}$ is the effective interaction fiber length and $\gamma$ is the nonlinear coefficient of the fiber. With the presence of the EDFA, the FWM noise power enhances significantly. In addition, the FWM noise power depends on the efficiency of the generated FWM wave, $n_{i}(f)$, given by [10]

$$
\begin{aligned}
n_{i}(f) & =\frac{\alpha^{2}}{\alpha^{2}+\left(\Delta \beta+2 \pi f d_{1, i}\right)} \\
& \times\left\{1+\frac{4 \exp (-\alpha L) \sin ^{2}\left[\frac{1}{2}\left(\Delta \beta+2 \pi f d_{1, i}\right) L\right]}{(1-\exp (-\alpha L))^{2}}\right\},
\end{aligned}
$$

Clearly, $n_{i}(f)$ depends on the phase matching condition that relates the propagation constant mismatch $\Delta \beta=\left(-\frac{D_{c} \lambda^{2}}{2 \pi c}\right)(2 \pi \Delta v)^{2}$ and the walkoff parameter $d_{1, i}$. The degeneracy factor $(D)$ can be six or three for nondegenerate FWM signals or degenerate ones, depending on $a_{d, i}$.

$$
a_{d, 1}=1 \text { for all }
$$

$$
\begin{aligned}
& a_{d, 2}= \begin{cases}1 & \text { for nondegenerate terms } \\
4 & \text { for degenerate terms }\end{cases} \\
& a_{d, 3}= \begin{cases}1 & \text { for nondegenerate terms } \\
0 & \text { for degenerate terms }\end{cases} \\
& a_{d, 4}= \begin{cases}1 & \text { for nondegenerate terms } \\
1 & \text { for degenerate terms }\end{cases}
\end{aligned}
$$

$$
a_{d, 5}= \begin{cases}2 & \text { for symmetric nondegenerate terms } \\ 0 & \text { for nonsymmetric nondegenerate } \\ & \text { and degenerate terms }\end{cases}
$$

In the photodiode, all the noise components comprising of shot noise, dark noise, and thermal noise contribute to the total noise of our proposed link. Thus, the photo-detector noise is defined as [9]

$$
N_{P D}=2 q B_{e}\left[R\left(P_{i n}+P_{\mathrm{ASE}}\right)+I_{d}\right]+S_{T}^{2} B_{e},
$$

where $q$ and $I_{d}$ are the electronic charge and the dark current, respectively. The ASE power caused by the presence of EDFA, $P_{\mathrm{ASE}}=S_{\mathrm{ASE}} B_{0} \exp (-\alpha L)$, makes the shot noise power increase. $P_{i n}$ is the total power on each optical channel at the input of the photodiode and can be estimated as $P_{\text {in }}=\left(P_{\mathrm{OFDM}}+P_{\mathrm{OC}}\right) G \exp (-\alpha L)$. The noise components from all parts of the OFDM WDM link analyzed above can be considered as independent Gaussian random variables [8-10], then the total noise after the photodiode can be expressed as

$$
N_{\text {total }}=N_{\mathrm{ACO}}+N_{\mathrm{EDFA}}+N_{\mathrm{FWM}}+N_{\mathrm{PD}} \text {. }
$$

\subsection{Wireless Channels}

Before being broadcasted to the wireless channel, the signal after PD is amplified by the power amplifier. In the wireless link, the signal mostly degraded due to free-space loss, atmospheric absorption, and rain attenuation [14-18]. Accordingly, the total loss of MMW link can be computed in decibel as

$$
\begin{aligned}
L_{\mathrm{wl}} & =L_{\mathrm{fs}}+L_{\mathrm{at}}+L_{\mathrm{rain}} \\
& =20 \log \frac{4 \pi d f_{\mathrm{mm}}}{c}+\left(\gamma_{\mathrm{ox}}+\gamma_{\mathrm{wv}}+\gamma_{\text {rain }}\right) d,
\end{aligned}
$$

where $L_{\mathrm{fs}}$ is free space path loss. $L_{\mathrm{at}}$ is the loss caused by atmospheric absorption oxygen and water vapor absorption. $L_{\text {rain }}$ is the attenuation due to rain. $d$ is the wireless link distance, $f_{\mathrm{mm}}$ is the MMW carrier frequency, and $c$ is the speed of light in vacuum. $\gamma_{\mathrm{ox}}, \gamma_{\mathrm{wv}}$, and $\gamma_{\text {rain }}$ are the attenuation coefficients induced by oxygen, water vapor, and rain, respectively.

In addition, MMW wireless channel can be modeled as either line-of-sight (LOS) or non-line-of-sight (NLOS) channel $[3,14]$. LOS MMW link requires highgain directional antennas thus mostly suffers from the path loss. NLOS MMW link without a strong direct LOS path is often modeled by Rayleigh distribution. Consequently, the probability density function (PDF) of the instantaneous SNR per bit $(\gamma)$ can be expressed as [19]

$$
P_{\gamma}(\gamma)=\frac{1}{\bar{\gamma}} \exp \left(-\frac{\gamma}{\bar{\gamma}}\right), \gamma \geq 0,
$$

where $\bar{\gamma}$ is the average SNR at the receiver.

\subsection{Bit Error Rate}

At the receiver located at $\mathrm{RRH}$, the received signal will be amplified by LNA before demodulated by OFDM demodulator. Therefore, signal to noise parameter of the wireless link at each receiver can be estimated as follows

$$
\bar{\gamma}=\frac{\left(R m P_{\mathrm{OFDM}} G e^{-\alpha L}\right)^{2} G_{P} G_{\mathrm{Tx}} G_{\mathrm{Rx}} / P_{L}}{N_{\text {total }} G_{P} G_{\mathrm{Tx}} G_{\mathrm{Rx}} / P_{L}+N F_{\mathrm{Rx}} K T B_{n}},
$$

where $G_{T x}$ and $G_{R x}$ are the gains of the transmitting and receiving antennas. $G_{P}$ and $G_{L}$ are the power gains of PA and LNA, respectively. $B_{n}$ is the effective 
noise bandwidth; $K$ is Boltzmann's constant; $T$ is the absolute temperature at the $\mathrm{RF}$ receiver; and $N F_{R x}$ is the receiving antenna noise figure.

The average bit error probability (i.e., BER) for the case of Rayleigh fading channel can be evaluated by the following integral [20]

$$
P_{b}^{R L}=\int_{0}^{\infty} P_{b}^{\mathrm{AWGN}}(\gamma) P_{\gamma}(\gamma) d \gamma
$$

where $P_{b}^{\mathrm{AWGN}}(\gamma)$ is the probability of error of a particular modulation scheme in non-fading AWGN channel. It is known that the BER for M-QAM in AWGN channel is written as [20]

$$
\begin{aligned}
P_{\mathrm{M}-\mathrm{QAM}}^{\mathrm{AWNG}}(\gamma) & =\frac{4}{\log _{2} M}\left(1-\frac{1}{\sqrt{M}}\right) \\
& \sqrt{M} / 2 \\
& \times \sum_{k=1} Q\left((2 k-1) \sqrt{\frac{3 \gamma \log _{2} M}{M-1}}\right) .
\end{aligned}
$$

In our analysis, 4-QAM is considered as it helps to keep RAU simple and does not require the SNR as high as that of $M$-QAM with higher modulation level such as 16-QAM or 64-QAM, which is not necessary for backhaul links. Consequently, BER could be rewritten as follows

$$
P_{4-\mathrm{QAM}}^{\mathrm{AWGN}}(\gamma)=Q(\sqrt{2 \gamma})=\frac{1}{2} \operatorname{erfc}(\sqrt{\gamma})
$$

where $Q(x)$ denotes Gaussian $Q$ function, which was given by Craig [20] and is defined as

$$
\begin{aligned}
Q(x) & =\frac{1}{\sqrt{2 \pi}} \int_{x}^{\infty} \exp \left(-\frac{t^{2}}{2}\right) d t \\
& =\frac{1}{\pi} \int_{0}^{\pi / 2} \exp \left(-\frac{x^{2}}{2 \sin ^{2} \theta}\right) d \theta
\end{aligned}
$$

From (11), (13), (15), (16), the bit error probability in Rayleigh fading channel can be expressed as [21]

$$
\begin{aligned}
P_{b}^{R L} & =\int_{0}^{\infty} \frac{1}{\pi} \int_{0}^{\pi / 2} \exp \left(-\frac{\gamma}{\sin ^{2} \theta}\right) d \theta \frac{1}{\bar{\gamma}} \exp \left(-\frac{\gamma}{\bar{\gamma}}\right) d \gamma \\
& =\frac{1}{\pi} \int_{0}^{\pi / 2}\left[\int_{0}^{\infty} \frac{1}{\bar{\gamma}} \exp \left(-\frac{\gamma}{\sin ^{2} \theta}\right) \exp \left(-\frac{\gamma}{\bar{\gamma}}\right) d \gamma\right] d \theta \\
& =\frac{1}{2}\left(1-\sqrt{\frac{\bar{\gamma}}{\bar{\gamma}+1}}\right) .
\end{aligned}
$$

For LOS MMW link, the MMW link mostly suffers from path loss, atmospheric absorption, and rain attenuation [16-19, 22]. Consequently, BER will be expressed as a function of average SNR for the case of M-QAM modulated data as follows [23]

$$
\mathrm{BER}=\frac{4}{\log _{2} M}\left(1-\frac{1}{\sqrt{M}}\right) Q\left(\sqrt{\frac{3}{M-1} \bar{\gamma}}\right)
$$

Table I

System Parameters And Constants

\begin{tabular}{lcl}
\hline \hline Name & Symbol & Value \\
\hline Fiber link & & \\
\hline Fiber attenuation coefficient & $\alpha$ & $0.2 \mathrm{~dB} / \mathrm{km}$ \\
Fiber nonlinear coefficient & $\gamma$ & $1.3(\mathrm{~W} \cdot \mathrm{km})^{-1}$ \\
Optical frequency of channel 1 & $v_{1}$ & $193.1 \mathrm{THz}$ \\
Channel spacing & $\Delta v$ & $200 \mathrm{GHz}$ \\
Dark current & $I_{d}$ & $0.1 \mathrm{nA}$ \\
Thermal noise power spectrum density & $S_{T}$ & $10^{-12} \mathrm{~A} / \mathrm{Hz}^{1 / 2}$ \\
PD responsivity & $R$ & $0.8 \mathrm{~A} / \mathrm{W}$ \\
The optical clipping power ratio & $\eta_{\mathrm{OCPR}}$ & $-5 \mathrm{~dB}$ \\
M-ary & $M$ & 4 \\
Data rate & $R_{b}$ & $10 \mathrm{Gbps}$ \\
\hline Wireless link & & \\
Wireless link distance & $d$ & $100 \mathrm{~m}$ \\
MMW frequency & $f_{\mathrm{mm}}$ & $60 \mathrm{GHz}$ \\
PA gain & $G_{P}$ & $35 \mathrm{~dB}$ \\
LNA gain & $G_{L}$ & $10 \mathrm{~dB}$ \\
Tx gain & $G_{\mathrm{Tx}}$ & $35 \mathrm{~dB}$ \\
Rx gain & $G_{\mathrm{Rx}}$ & $30 \mathrm{~dB}$ \\
Implementation loss & $P_{I}$ & $6 \mathrm{~dB}$ \\
Rx noise figure & $N F_{\mathrm{Rx}}$ & $6.53 \mathrm{~dB}$ \\
\hline \hline
\end{tabular}

\section{Numerical Results}

Based on the expressions obtained in Section 3, in this section, the BER performance of the OFDM RoF-based WDM-PON/MMW downlink is investigated with different system parameters consisting of the launched optical power of each channel, the modulation index, and EDFA. As this backhaul downlink is based on WDM-PON, the fiber's maximum physical transmission reach of $40 \mathrm{~km}$ is chosen for our analysis. The channel spacing in optical frequency is $200 \mathrm{GHz}$ for transmitting double sideband signal with RF subcarrier of $60 \mathrm{GHz}$. In addition, two wireless channel models including LOS and NLOS are taken into account. Other system parameters and constants used in our analysis are shown in Table I.

In Figure 3, BER of the proposed downlink is demonstrated versus the launched optical power for the case of using standard single-mode fiber (SMF) with $D_{c}=17$ ps/(nm.km). Two types of wireless connections considered in this result are NLOS and LOS. For the cases of NLOS connection, because of the influence of multipath fading, the BER is higher than $10^{-3}$ thus system performance does not meet the requirement. The BER performance for the cases of LOS connections can achieve the values smaller than $10^{-6}$, which is acceptable when forward error correction (FEC) code is not used. It is noted that the system with BER of $10^{-6}$ can guarantee error-free when FEC is employed.

The impacts of FWM and noise components are also clearly shown in Figure 3. More specifically, when the launched power is smaller than a specific value, the impact of noise components such as clipping noise, EDFA noise, and the PD noise are dominant compared to the impact of FWM. As a result, the increase of launched power helps to reduce BER. However, the impact of FWM increases significantly when the launched power is larger than a specific value. Accordingly, there is an 


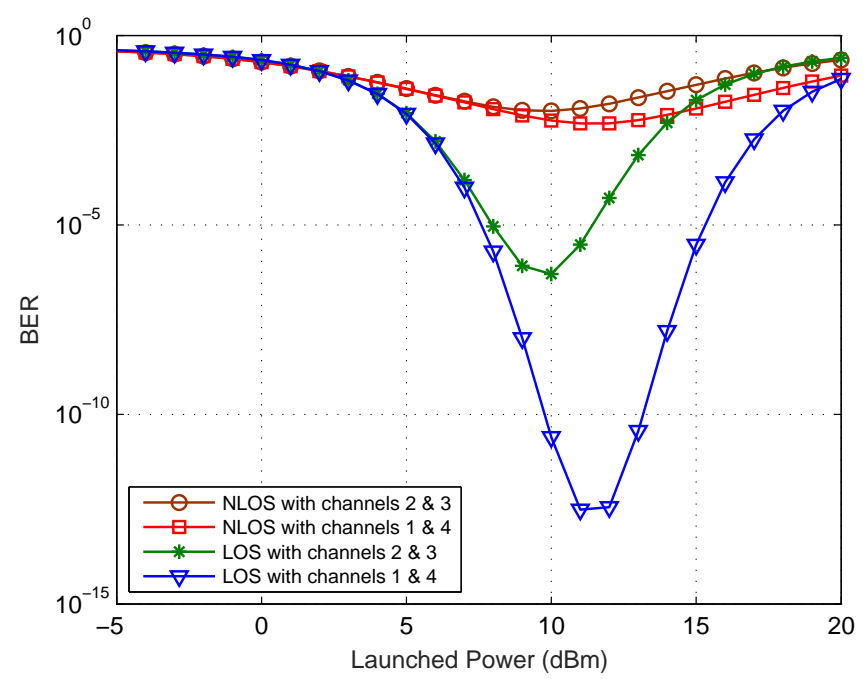

Figure 3. BER versus the launched optical power with SMF, EDFA gain $G=10 \mathrm{~dB}$, and modulation index $m=0.025$.

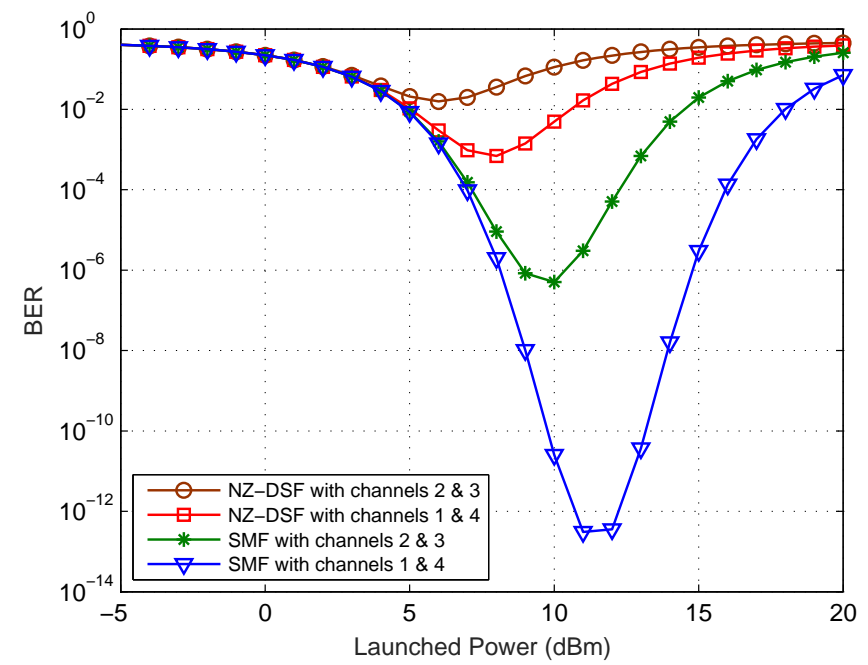

Figure 4. BER versus the launched power with SMF and NZ-DSF, LOS connection, EDFA gain $G=10 \mathrm{~dB}$, and modulation index $m=$ 0.025 .

optimum value of launched optical power, where the link's BER is minimized. The figure also shows that the impact of FWM on each wavelength (or channel) in WDM link is different. BER of the central channels (i.e., channels 2 and 3 ) is deteriorated more significantly compared to that of the edge channels (i.e., channels 1 and 4).

Figure 4 shows the BER curves versus the launched optical power for two different types of optical fiber including SMF and non-zero dispersion-shifted fiber (NZ-DSF) with $D_{c}=4 \mathrm{ps} /(\mathrm{nm} . \mathrm{km})$. It is well known that NZ-DSF is designed to overcome the dispersion problem, it is however more affected by FWM. Consequently, the system using NZ-DSF has BER larger than that of the one using SMF. This is due to the fact that the small value of dispersion parameter in NZDSF results in the large impact of FWM. Therefore, it is recommended that NZ-DSF should not be employed

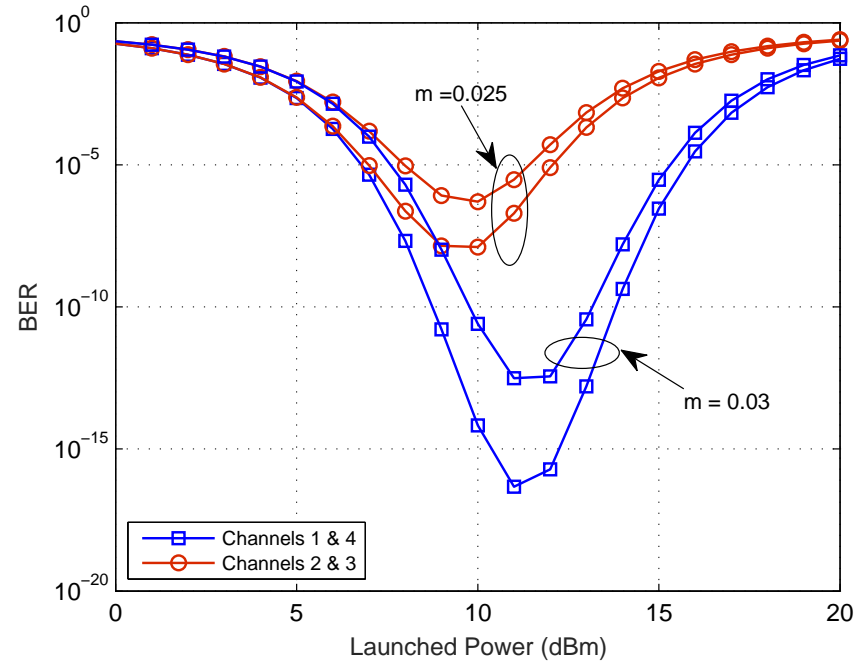

Figure 5. BER versus the optical launched power with SMF, LOS connection and EDFA gain $G=10 \mathrm{~dB}$.

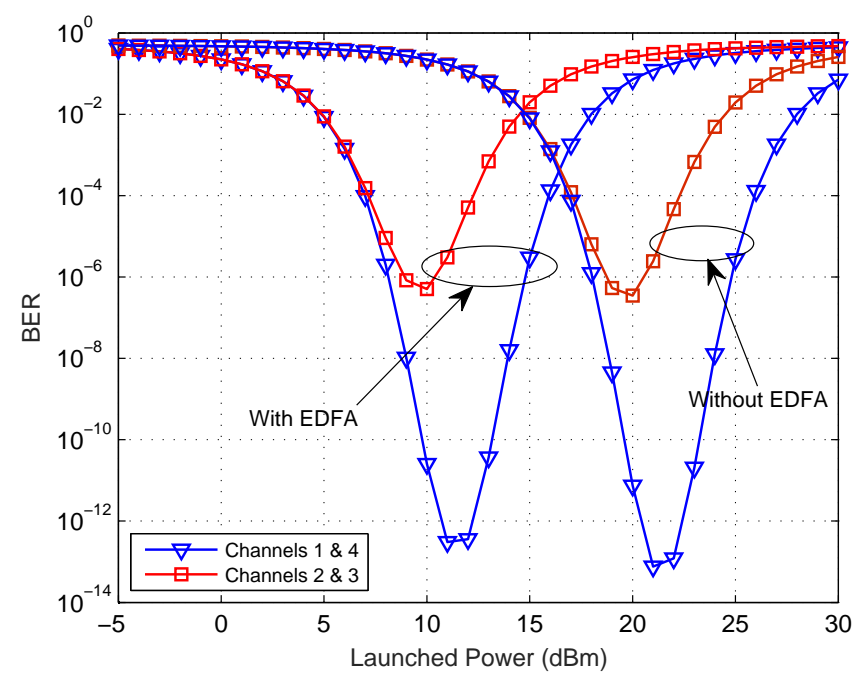

Figure 6. BER versus the optical launched power with SMF, LOS connection, and $m=0.025$.

in OFDM RoF-based WDM-PON.

In Figure 5, the BER of the proposed downlink is investigated with different modulation indexes, which governs the impact of clipping noise. The system using SMF with launched power of $0 \mathrm{dBm}$ and EDFA gain of $10 \mathrm{~dB}$ is used. The increase in modulation index (from 0.025 to 0.03 ) helps to increase the signal power hence reduce the BER. However, high modulation index causes the increase in clipping noise. As a result, there should be an optimum modulation index at which the best performance can be obtained.

Next, Figure 6 presents the difference in the BER of the proposed downlink for the case of with and without using EDFA. When an EDFA with the gain of $10 \mathrm{~dB}$ is used, all the curves are shifted to the left correspondingly $10 \mathrm{~dB}$ compared to the case of without using EDFA. More specifically, the required high launched power can be relieved by the use of 


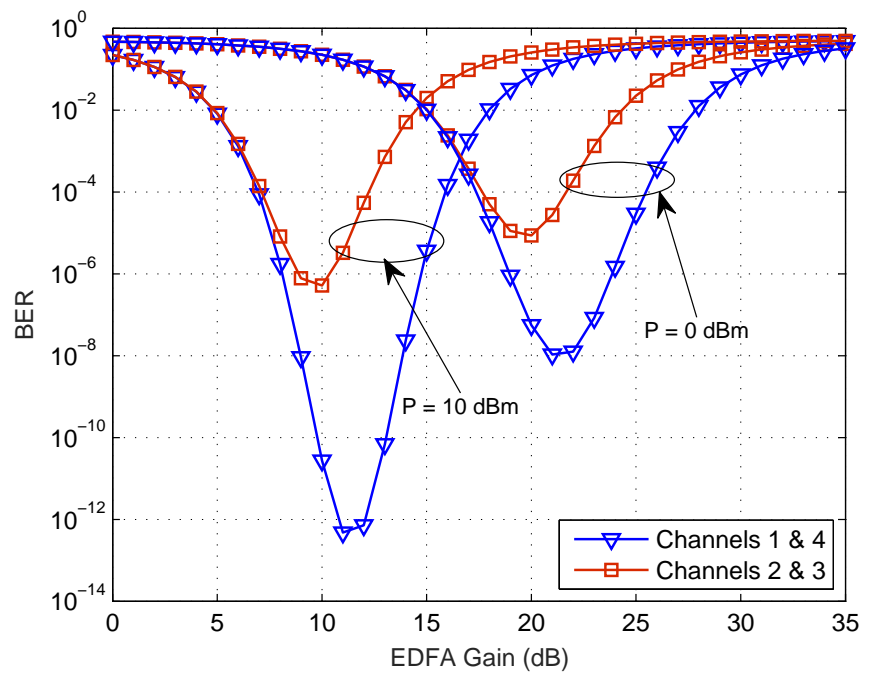

Figure 7. BER versus the EDFA gain with the launched optical power $0 \mathrm{dBm}$ and $10 \mathrm{dBm}, \mathrm{SMF}$ with LOS connection, and $m=0.025$.

EDFA hence, the expensive high-power light sources are not required in the CS. However, EDFA adds the amplified spontaneous emission noise to the system that makes the overall BER performance deteriorate slightly compared to the BER performance for the case of without using EDFA.

Finally, in Figure 7, the impact of the EDFA gain $(G)$ on the link's BER is investigated with the launched optical power of $0 \mathrm{dBm}$ and $10 \mathrm{dBm}$. Clearly, EDFA gain should be chosen properly to obtain the best performance since it not only help to increase the launched optical power but also causes the increase in ASE noise and FWM noise. These noise components become dominant when EDFA gain is larger than a specific value. Consequently, the increase of BER can be seen for all channels. The degradation level of the BER also depends on the values of the launched optical power. More specifically, the optimum EDFA gain at the launched optical power of $10 \mathrm{dBm}$ is smaller than that of $0 \mathrm{dBm}$. However, the BER at the launched optical power of $0 \mathrm{dBm}$ is degraded compared to that at 10 $\mathrm{dBm}$. This is because lower launched optical power requires higher EDFA gain, which induces larger ASE noise and FWM noise. Actually, the power of FWM noise increases with $G^{4}$ while the power of ASE noise is in proportional to $G^{2}$.

\section{Conclusion}

We have proposed a hybrid OFDM RoF-based WDMPON/MMW architecture for heterogeneous wireless networks. The performance of the downlink in the proposed networks was investigated under the influence of fiber nonlinear, wireless fading, and noise components including clipping noise, amplifier noise and PD noise. The impact of these physical layer impairments was quantified in different scenarios for both the optical fiber types and the wireless channel models. The numerical results have proven the feasibility of our pro- posed backhaul downlink architecture in terms of performance for the case of LOS connection. For the cases of NLOS connection, the system performance does not meet the requirement mainly due to the influence of multipath fading.

The system parameters such as the launched power, modulation index, and EDFA gain play an important role in the overall performance of our proposed architecture thus they should be chosen properly in order to minimize the BER. The value of the parameters obtained from the numerical results would be useful information for designing a backhaul link in heterogeneous wireless networks. For instance, the launched power should be in around $10 \mathrm{dBm}$ with the amplifier gain of $10 \mathrm{~dB}$ in order to establish a hybrid OFDM RoF-based WDM-PON/MMW downlink with the 40$\mathrm{km}$ fiber link and wireless link distance of $100 \mathrm{~m}$. The system with more than four wavelengths will be more affected by FWM hence methods for mitigating FWM should be applied such as increasing the channel spacing or using unequal-spaced channel allocation techniques. A similar approach can be used for analyzing the performance of the uplink. In the case that the system is symmetrical, the system's parameters are the same for both the downlink and uplink.

\section{ACKNOWLEDGMENT}

This research is funded by Vietnam National Foundation for Science and Technology Development (NAFOSTED) under the grant number 102.02-2015.06.

\section{REFERENCES}

[1] T. S. Rappaport, S. Sun, R. Mayzus, H. Zhao, Y. Azar, K. Wang, G. N. Wong, J. K. Schulz, M. Samimi, and F. Gutierrez, "Millimeter wave mobile communications for 5G cellular: It will work!" IEEE access, vol. 1, pp. 335349, 2013.

[2] C.-X. Wang, F. Haider, X. Gao, X.-H. You, Y. Yang, D. Yuan, H. Aggoune, H. Haas, S. Fletcher, and E. Hepsaydir, "Cellular architecture and key technologies for 5G wireless communication networks," IEEE Communications Magazine, vol. 52, no. 2, pp. 122-130, 2014.

[3] X. Ge, H. Cheng, M. Guizani, and T. Han, "5G wireless backhaul networks: Challenges and research advances," IEEE Network, vol. 28, no. 6, pp. 6-11, 2014.

[4] H. Le Bras and M. Moignard, "Distribution of 3G base stations on passive optical network architecture," in Proceedings of the International Topical Meeting on Microwave Photonics (MWP'06). IEEE, 2006, pp. 1-4.

[5] E. Mallette, "Mobile backhaul for PON: A case study," in Proceedings of the Optical Fiber Communication Conference and Exposition and the National Fiber Optic Engineers Conference (OFC/NFOEC). IEEE, 2012, pp. 1-3.

[6] V. K. Pandey, S. Gupta, and B. Chaurasiya, "Performance Analysis of WDM PON and ROF Technology in Optical Communication Based on FBG," International Journal of Engineering Research, vol. 3, no. 10, pp. 608-612, 2014.

[7] T. Shao, F. Paresys, Y. Le Guennec, G. Maury, N. Corrao, and B. Cabon, "Convergence of $60 \mathrm{GHz}$ radio over fiber and WDM-PON using parallel phase modulation with a single Mach-Zehnder modulator," Journal of lightwave technology, vol. 30, no. 17, pp. 2824-2831, 2012. 
[8] S. Dimitrov and H. Haas, "On the clipping noise in an ACO-OFDM optical wireless communication system," in Proceedings of the Global Telecommunications Conference (GLOBECOM 2010). IEEE, 2010, pp. 1-5.

[9] G. P. Agrawal, Fiber-Optic Communication Systems, 3rd ed., ser. ISBNs: 0-471-21571-6 (Hardback); 0-47122114-7 (Electronic). John Wiley \& Sons, 2002, vol. 222.

[10] T. M. Alves and A. V. Cartaxo, "Analytical characterization of four wave mixing effect in direct-detection double-sideband OFDM optical transmission systems," Optics Express, vol. 22, no. 7, pp. 8598-8616, 2014.

[11] J. Armstrong, B. J. Schmidt, D. Kalra, H. A. Suraweera, and A. J. Lowery, "SPC07-4: Performance of asymmetrically clipped optical OFDM in AWGN for an intensity modulated direct detection system," in Proceedings of the Global Telecommunications Conference (GLOBECOM'06). IEEE, 2006, pp. 1-5.

[12] D. Uzunidis, C. Matrakidis, and A. Stavdas, "Simplified model for nonlinear noise calculation in coherent optical OFDM systems," OSA Optics Express, vol. 22, no. 23, pp. 28316-28326, 2014.

[13] G. P. Agrawal, Nonlinear Fiber Optics, 3rd ed. Academic Press, 2001.

[14] M. Cvijetic and I. Djordjevic, Advanced Optical Communication Systems and Networks. Artech House Applied Photonics, 2013.

[15] T. A. Pham, H. T. Pham, L. T. Vu, and N. T. Dang, "Effects of noise and distortion on performance of OFDM millimeter-wave RoF systems," in Proceedings of the 2nd National Foundation for Science and Technology Development Conference on Information and Computer Science (NICS). IEEE, 2015, pp. 153-157.

[16] S. Rajagopal, S. Abu-Surra, and M. Malmirchegini, "Channel feasibility for outdoor non-line-of-sight mmwave mobile communication," in Proceedings of the Vehicular Technology Conference (VTC Fall). IEEE, 2012, pp. 1-6.

[17] J. Qiao, X. S. Shen, J. W. Mark, Q. Shen, Y. He, and L. Lei, "Enabling device-to-device communications in millimeter-wave 5G cellular networks," IEEE Communications Magazine, vol. 53, no. 1, pp. 209-215, 2015.

[18] C. Gustafson, "60 GHz wireless propagation channels: Characterization, modeling and evaluation," Ph.D. dissertation, Lund University, 2014.

[19] M. Kyrö, "Radio wave propagation and antennas for millimeter-wave communications," Aalto University publication series, Doctoral dissertation, 2012.

[20] M. K. Simon and M.-S. Alouini, Digital Communication over Fading Channels. John Wiley \& Sons, 2005, vol. 95.

[21] T. A. Pham, H. T. Pham, H.-C. Le, and N. T. Dang, "Numerical analysis of the performance of millimeterwave RoF-based cellular backhaul links," Journal of Optical Communications, vol. 38, no. 3, pp. 309-318, 2017.

[22] Y.-T. Hsueh, "Frontiers of optical networking technologies: Millimeter-wave radio-over-fiber and 100G transport system for next-generation high-data-rate applications," Ph.D. dissertation, Georgia Institute of Technology, 2012.

[23] J. G. Proakis, Digital Communications, 3rd ed. McGrawHill: Singapore, 1995.

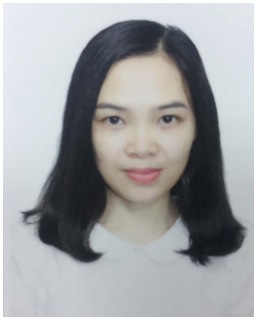

Trang Thi Thu Ngo received B.E degree of Telecommunications and Electronics Engineering from Vietnam National University, Hanoi (VNUH) in 2002, and M.E. degree of Computer and Communication Engineering from Chungbuk National University, Korea in 2005. Now, she is a lecturer and Ph.D. student in Telecommunications Faculty of Posts and Telecommunications Institute of Technology (PTIT). Her research interests include optiand broadband networks. cal communication, digital signal processing,

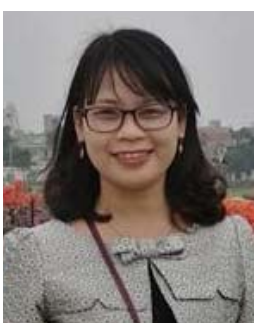

Thu Anh Pham received B.E degree of Telecommunication engineering from Posts and Telecommunications Institute of Technology (PTIT), Vietnam, in 2003, and M.E. degree of Telecommunication engineering from Royal Melbourne Institute of Technology, Australia, in 2008. Now, she is a lecturer and Ph.D. student in Telecommunication faculty of PTIT. Her research interests include networking, radio over fiber, and broadband networks.

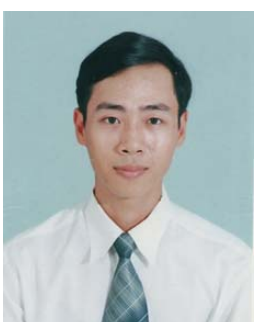

Nhan Duc Nguyen received the B.Sc. in chemistry from the Vietnam National University, Hanoi in 1996 and M.Eng. in electronic materials from International Training Institute for Materials Science (ITIMS), Hanoi University of Technology in 1998. In 1999 he joined the Department of Optical Communication, Faculty of Telecommunication, Post and Telecommunication Institute of Technology in Vietnam as a lecturer. He received Ph.D. degree in electrical engineering and computer systems from Monash University, Australia in 2011. Since then he has been with the Faculty of Telecommunication, Post and Telecommunication Institute of Technology. His research interests are numerical modeling and analysis, signal processing and its applications in optical communications and nonlinear fiber optics applications.

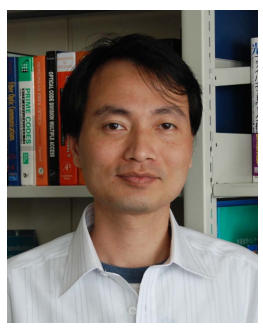

Ngoc The Dang received the B.E. degree from the Hanoi University of Technology, Hanoi, Vietnam in 1999, and the M.E. degree from the Posts and Telecommunications Institute of Technology (PTIT), Hanoi, Vietnam in 2005, both in electronics and telecommunications; and received the Ph.D. degree in computer science and engineering from the University of Aizu, Aizuwakamatsu, Japan in 2010. He is currently an Associate Professor/Head with the Department of Wireless Communications at PTIT. He was also an invited researcher at FOTON-ENSSAT Lab., Universite de Rennes 1, (France) in 2011 and a research fellow at Computer Communications Lab., The University of Aizu (Japan) in 2012, 2013, 2015, and 2017. His current research interests include the area of communication theory with a particular emphasis on modeling, design, and performance evaluation of optical CDMA, RoF and optical wireless communication systems. 